\title{
Oxidative Stress and Mitochondrial DNA Damage in Heart Failure
}

\author{
Hiroyuki Tsutsui, MD; Shintaro Kinugawa, MD; Shouji Matsushima, MD
}

\begin{abstract}
Recent experimental and clinical studies have suggested that oxidative stress is enhanced in heart failure. The production of oxygen radicals is increased in the failing heart while antioxidant enzyme activities are preserved. Mitochondrial electron transport is an enzymatic source of oxygen radical generation and also a target against oxidant-induced damage in the failing myocardium. Chronic increases in oxygen radical production in the mitochondria can lead to a catastrophic cycle of mitochondrial DNA (mtDNA) damage, as well as functional decline, further oxygen radical generation, and cellular injury. Reactive oxygen species induce myocyte hypertrophy, apoptosis, and interstitial fibrosis by activating matrix metalloproteinases. These cellular events play an important role in the development and progression of maladaptive cardiac remodeling and failure. Therefore, oxidative stress and mtDNA damage are good therapeutic targets. Overexpression of peroxiredoxin-3 (Prx-3), mitochondrial antioxidant, or mitochondrial transcription factor A (TFAM) could ameliorate the decline in mtDNA copy number in failing hearts. Consistent with alterations in mtDNA, the decrease in oxidative capacities is also prevented. Therefore, the activation of Prx-3 or TFAM expression could ameliorate the pathophysiological processes seen in myocardial failure. Inhibition of oxidative stress and mtDNA damage could be novel and potentially effective treatment strategies for heart failure. (Circ J 2008; Suppl A: A-31-A-37)
\end{abstract}

Key Words: DNA; Heart failure; Mitochondria; Oxidative stress; Remodeling

$\mathbf{H}$ eart failure is a leading cause of morbidity and mortality in industrialized countries! It is also a growing public health problem, mainly because of aging of the population and the increase in the prevalence of heart failure in the elderly. Previous basic, clinical, and population sciences have advanced the modern treatment of heart failure, but despite extensive studies, the fundamental mechanisms responsible for the development and progression of left ventricular (LV) failure have not yet been fully elucidated.

Reactive oxygen species (ROS), such as superoxide anions $\left(\cdot \mathrm{O}_{2}-\right)$ and hydroxy radicals $(\cdot \mathrm{OH})$, cause the oxidation of membrane phospholipids, proteins, and $\mathrm{DNA}^{2}$ and have been implicated in a wide range of pathological conditions including ischemia-reperfusion injury? neurodegenerative diseases, and aging5. Under physiological conditions, their toxic effects can be prevented by such scavenging enzymes as superoxide dismutase (SOD), glutathione peroxidase (GSHPx), and catalase, as well as by other non-enzymatic antioxidants. However, when the production of ROS becomes excessive, oxidative stress might have a harmful effect on the functional and structural integrity of biological tissue.

ROS cause contractile failure and structural damage in the myocardium. The importance of oxidative stress is in-

(Received January 15, 2008; accepted June 22, 2008; released online September 4, 2008)

Department of Cardiovascular Medicine, Hokkaido University Graduate School of Medicine, Sapporo, Japan

Mailing address: Hiroyuki Tsutsui, MD, Department of Cardiovascular Medicine, Hokkaido University Graduate School of Medicine, Kita-15, Nishi-7, Kita-ku, Sapporo 060-8638, Japan. E-mail: htsutsui @ med.hokudai.ac.jp

All rights are reserved to the Japanese Circulation Society. For permissions, please e-mail: cj@j-circ.or.jp creasingly emerging, with respect to a pathophysiological mechanism of the LV remodeling responsible for heart failure progression.

\section{Direct Evidence of Oxidative Stress in Heart Failure}

Recent experimental and clinical studies have suggested that the generation of ROS increases in heart failure ${ }^{6-9}$ Lipid peroxides and 8-iso-prostaglandin F2 $\alpha$, which are the major biochemical markers of ROS generation, have been shown to be elevated in the plasma and pericardial fluid of patients with heart failure and also positively correlated with severity 6,9

Using electron spin resonance (ESR) spectroscopy combined with the nitroxide radical, 4-hydroxy-2,2,6,6-tetramethyl-piperidine-N-oxyl, a definitive and direct demonstration of enhanced generation of ROS in the failing myocardium has been obtained $!^{10}$ The $\cdot \mathrm{O}_{2}{ }^{-}$is a primary radical that could lead to the formation of other ROS, such as $\mathrm{H}_{2} \mathrm{O}_{2}$ and $\cdot \mathrm{O}_{2}{ }^{-}$, in the failing myocardium. The $\cdot \mathrm{OH}$ could arise from electron exchange between ${ }^{\circ} \mathrm{O}_{2}-$ and $\mathrm{H}_{2} \mathrm{O}_{2}$ via the Harber-Weiss reaction. In addition, $\cdot \mathrm{OH}$ is generated by the reduction of $\mathrm{H}_{2} \mathrm{O}_{2}$ in the presence of endogenous iron by means of the Fenton reaction. The generation of $\cdot \mathrm{OH}$ implies a pathophysiological significance of ROS in heart failure, because .OH radicals are the predominant oxidant species causing cellular injury.

Decreased antioxidant capacity could further aggravate ROS accumulation in heart failure; however, the activities of SOD, catalase, and GSHPx are not decreased in the failing heart 11 indicating that oxidative stress in heart failure is primarily related to enhancement of pro-oxidant generation rather than to a decline in antioxidant defenses. 


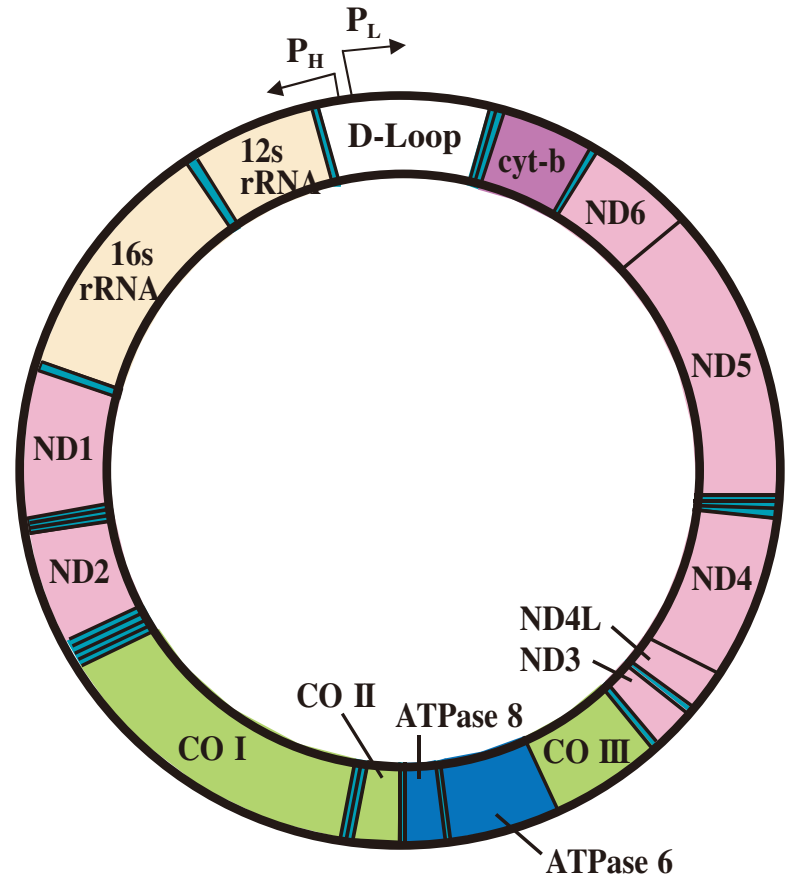

\section{Mitochondria as a Source of Oxidative Stress}

The cellular sources of ROS generation within the heart include cardiac myocytes, endothelial cells, and neutrophils. Within cardiac myocytes, ROS can be produced by several mechanisms, including mitochondrial electron transport, NADPH oxidase, and xanthine dehydrogenase/xanthine oxidase.

The heart has the highest oxygen uptake rate within the human body, consuming about $0.1 \mathrm{ml} \mathrm{O}_{2} / \mathrm{g} \mathrm{min}$ at basal rates. To meet the demand for synthesis of ATP by oxidative metabolism, cardiac myocytes have the highest volume density of mitochondria, which produce ROS through 1-electron carriers in the respiratory chain. Under physiological conditions, the small quantities of ROS formed during mitochondrial respiration can be detoxified by the endogenous scavenging mechanisms of myocytes.

Using ESR spectroscopy with 5,5'-dimethyl-1-pyrroline$\mathrm{N}$-oxide as a spin trap, the inhibition of electron transport at the sites of complex I and complex III in the normal submitochondrial particles results in a significant production of $\cdot \mathrm{O}_{2}-12$ Mitochondria in heart failure produce more $\cdot \mathrm{O}_{2}{ }^{-}$ than normal mitochondria in the presence of NADH, indicating that mitochondrial electron transport could be the predominant source of such $\mathrm{O}_{2}{ }^{-}$production. Furthermore, the failing mitochondria are associated with a decrease in complex enzyme activity. Therefore, mitochondria are an important source of ROS in the failing heart, indicating a pathophysiological link between mitochondrial dysfunction and oxidative stress ${ }^{13}$ as has been reported in other disease conditions including aging and neurodegenerative diseases.

Even though mitochondrial electron transport is considered to play an important role in ROS production in heart failure, we can not completely exclude the possibility that other enzymatic sources of ROS generation, such as vascular endothelial cells (via xanthine oxidase and/or NADPH oxidase) and activated leukocytes (via NADPH oxidase), also contribute to oxidative stress ${ }^{14}$ In fact, Bauersachs et al have demonstrated that vascular $\mathrm{NAD}(\mathrm{P}) \mathrm{H}$ oxidase is acti-

\section{Complex I \\ Complex III \\ Complex IV \\ Complex V \\ tRNA}

Fig 1. Map of the mitochondrial genome. The $16.3-\mathrm{kb}$ mouse mitochondrial genome is shown with the 13 mRNA, 2 rRNA ( $12 \mathrm{~S}$ and $16 \mathrm{~S}$ ), and 21 tRNA coding genes. mRNA genes are the areas labeled with the codes of the corresponding electron transport chain complexes I, III, IV, and $\mathrm{V}$. PH and PL are the promoters of heavy $(\mathrm{H})$ and light $(\mathrm{L})$ strand transcription, respectively.

vated in heart failure 15 This enzyme system is the major source of ROS in both the endothelium and vascular smooth muscle, which are able to generate ROS in response to angiotensin II and thus stimulate the expression of $\mathrm{NAD}(\mathrm{P}) \mathrm{H}$ oxidase. Plasma renin activity, as well as tissue angiotensinconverting enzyme activity, is activated in heart failure. Therefore, enhanced formation of angiotensin II may lead to oxidative stress via this enzyme system.

\section{Consequences of Oxidative Stress in Heart Failure}

\section{Oxidative Stress and Mitochondrial DNA (mtDNA) Damage}

Mitochondria have their own genomic system, mtDNA, a closed-circular double-stranded DNA molecule of approximately $16.5 \mathrm{~kb}$ (Fig 1). MtDNA contains 2 promoters, the light-strand and heavy-strand promoters (LSP and HSP, respectively), from which transcripts are produced and then processed to yield the individual mRNAs encoding 13 subunits of the oxidative phosphorylation, including 7 subunits (ND1, ND2, ND3, ND4, ND4L, ND5, and ND6) of rotenone-sensitive NADH-ubiquinone oxidoreductase (complex I), 1 subunit (cytochrome b) of ubiquinol-cytochrome c oxidoreductase (complex III), 3 subunits (COI, COII, and COIII) of cytochrome-c oxidase (complex IV), and 2 subunits (ATPases 6 and 8) of complex V, together with 22 tRNAs and 2 rRNA (12S and 16S) subunits! 16,17 Transcription from the LSP also produces RNA primer, which is necessary for initiating mtDNA replication. Mitochondrial function is controlled by the mtDNA, as well as factors that regulate mtDNA transcription and/or replication! ${ }^{18}$ This raises the possibility that mitochondrial gene replication, and thus the mtDNA copy number and/or mitochondrial gene transcription, are impaired in heart failure. Indeed, heart failure is frequently associated with qualitative and quantitative defects in mtDNA ${ }^{19-22}$ Recently, the decline in mitochondrial function and mtDNA copy number was shown to play a major role in the development of the heart failure that 


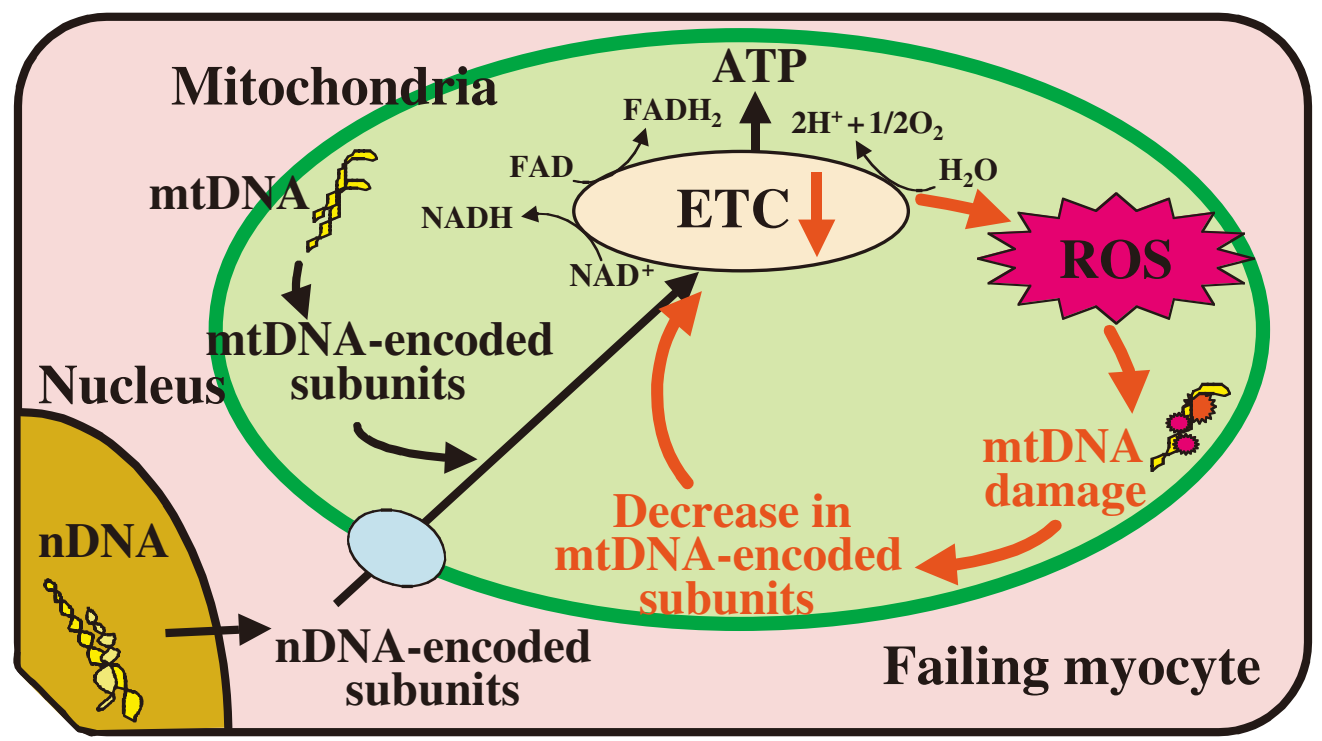

Fig 2. Schematic representation of the intimate link between reactive oxygen species (ROS), mitochondrial DNA (mtDNA) damage, and respiratory chain dysfunction in the mitochondria. Mitochondrial ROS generation may lead to a catastrophic cycle of mitochondrial functional decline, further ROS generation, and cellular injury.

occurs after myocardial infarction (MI) ${ }^{12,23}$

ROS can damage mitochondrial macromolecules either at or near the site of their formation. Therefore, in addition to the role of mitochondria as a source of ROS, the mitochondria themselves can be damaged by ROS. The mtDNA could be a major target for ROS-mediated damage for several reasons. First, mitochondria do not have the complex chromatin organization consisting of histone proteins that may serve as a protective barrier against ROS. Second, mtDNA has limited repair ability against DNA damage. Third, a large part of the ${ }^{-\mathrm{O}_{2}}{ }^{-}$formed inside the mitochondria can not pass through the membranes and hence, ROS damage may be contained largely within the mitochondria. In fact, mtDNA accumulates significantly higher levels of the DNA oxidation product, 8-hydroxydeoxyguanosine, than nuclear DNA24 As opposed to nuclear-encoded genes, mitochondrial-encoded gene expression is largely regulated by the copy number of mtDNA. ${ }^{25}$ Therefore, mitochondrial injury is reflected by mtDNA damage, as well as by a decline in the mitochondrial RNA (mtRNA) transcripts, protein synthesis, and mitochondrial function 26,27 We have shown that increased generation of ROS is associated with mitochondrial damage and dysfunction in the failing heart, characterized by increased lipid peroxidation in the mitochondria, decreased mtDNA copy number, a decrease in the number of mtRNA transcripts, and reduced oxidative capacity because of low complex enzyme activities ${ }^{23} \mathrm{~A}$ chronic increase in ROS production is associated with mitochondrial damage and dysfunction, which can lead to a catastrophic cycle of mitochondrial functional decline, further ROS generation, and cellular injury (Fig 2). MtDNA defects may thus play an important role in the development and progression of myocardial remodeling and failure.

A number of pathogenic mtDNA base substitution mutations, such as missense mutations and mtDNA rearrangement mutations (deletions and insertions), have been identified in patients with mitochondrial diseases 22 An accumulation of the deleted forms of mtDNA in the myocardium frequently results in cardiac hypertrophy, conduction block, or heart failure ${ }^{28}$ Furthermore, there is now a consensus view that mutations in mtDNA and abnormalities in mitochondrial function are associated with common forms of cardiac disease, such as ischemic heart disease ${ }^{29}$ and dilated cardiomyopathy $3^{30}$ In those conditions, however, the strict causal relationships between abnormalities in mtDNA and cardiac dysfunction have yet to be fully elucidated ${ }^{31}$ Even though the mechanisms by which mtDNA damage arises have not been clarified, ROS have been proposed as the primary contributing factor. We have provided direct evidence that mtDNA defects occur not only in a limited, small subset of mitochondrial diseases, but also in a more common form of heart failure phenotype occurring after MI.

\section{Oxidative Stress and Myocardial Damage}

ROS have direct effects on cellular structure and function, and may be integral signaling molecules in myocardial remodeling and failure. ROS result in a phenotype characterized by hypertrophy and apoptosis in isolated cardiac myocytes 32 ROS have also been shown to activate matrix metalloproteinase (MMP) in cardiac fibroblasts 33 Myocardial MMP activity is increased in the failing heart:32,34 Furthermore, an MMP inhibitor has been shown to limit early LV dilatation in a murine model of MI ${ }^{35}$ We have shown significant improvement in survival after MI in MMP-2 knockout mice, which was mainly attributable to inhibition of early cardiac rupture and the development of subsequent LV dysfunction? ${ }^{36}$ Because MMP can be activated by ROS $3{ }^{37}$ a proposed mechanism of LV remodeling is activation of MMPs secondary to increased ROS production. Sustained MMP activation might influence the structural properties of the myocardium by providing an abnormal extracellular environment with which the myocytes interact. We have demonstrated that the $\cdot \mathrm{OH}$ scavenger, dimethylthiourea, inhibits the activation of MMP-2 in association with the development of $\mathrm{LV}$ remodeling and failure ${ }^{38}$ These findings raise the interesting possibility that increased ROS after MI may be a stimulus for myocardial MMP activation, which 
Mitochondria
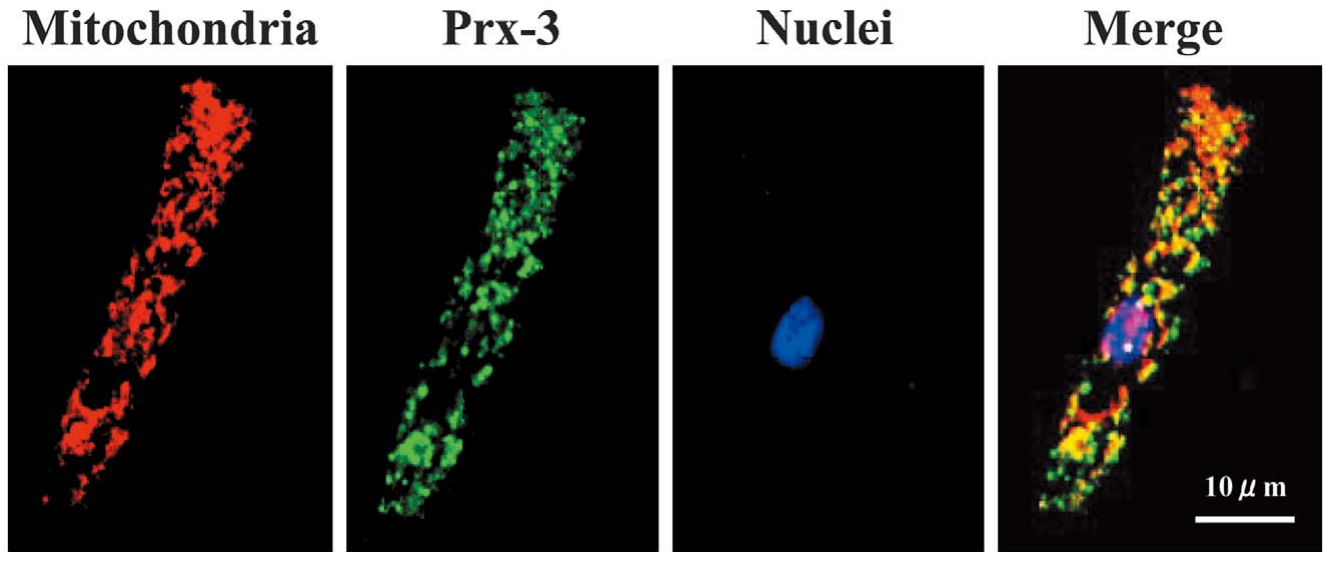

Fig 3. Cardiac myocytes isolated from transgenic mice were doubly stained with MitoTracker dye (red), a rat Prx-3specific antibody (green), and nucleus (blue). Immunoreactivity for Prx-3 can be seen in the cytoplasm of cardiac myocytes. The merged images show Prx-3 colocalized with the mitochondria (yellow). Scale bar $=10 \mu \mathrm{m}$.

then play an important role in the development of heart failure.

\section{Oxidative Stress and Skeletal Muscle Damage}

Limited exercise capacity is a major symptom in patients with heart failure ${ }^{39}$ and is independent of the degree of cardiac dysfunction. ${ }^{40}$ Increased oxidative stress has been shown to be related to the limitation of exercise capacity in patients with heart failure 41 We have demonstrated that ROS are increased in skeletal muscle in patients with heart failure after $\mathrm{MI}$ and that they originate from $\cdot \mathrm{O}_{2}-$ produced by mitochondrial oxidase? 42 Recently, Kinugawa et al clarified the relationship between $\mathrm{O}_{2}{ }^{-}$and the limitation of exercise capacity by using heterozygous manganese superoxide anion dismutase (SOD2) gene-knockout mice, in which SOD2, a family of enzymes that catalyze the dismutation of $\cdot \mathrm{O}_{2}{ }^{-}$, is reduced by $30-80 \%$, increasing $\cdot \mathrm{O}_{2}-$ production in the mitochondria, associated with altered mitochondrial function 43 The whole-body oxygen consumption $\left(\dot{\mathrm{VO}}_{2}\right)$ and carbon dioxide production $\left(\dot{\mathrm{V}} \mathrm{CO}_{2}\right)$ at rest were increased in SOD2 ${ }^{+/}$. The work (vertical distance run $\times$body weight) to exhaustion was decreased in SOD2+/-. When the maximum $\dot{\mathrm{V}} \mathrm{O}_{2}$ and $\dot{\mathrm{V}} \mathrm{CO}_{2}$ were corrected to the per work unit, they were increased in SOD2 ${ }^{+/}$. Tempol normalized the basal $\dot{\mathrm{V}} \mathrm{O}_{2}$ and $\dot{\mathrm{V}} \mathrm{CO}_{2}$ and improved the work to exhaustion and corrected $\dot{\mathrm{V}} \mathrm{O}_{2}$ and $\dot{\mathrm{V} C O} 2$ in $\mathrm{SOD}_{2}{ }^{+/}$. There was a decrease in the SOD2 protein level and a concomitant increase in lucigenin-detectable $\cdot \mathrm{O}_{2}-$ production in skeletal muscle from SOD2+/-. Therefore, exercise capacity was reduced in conditions in which $\mathrm{O}_{2}{ }^{-}$was increased, and this was associated with a greater increase in whole-body oxygen consumption.

\section{Amelioration of Oxidative Stress, MtDNA Damage, and Heart Failure}

\section{GSHPx}

The first line of defense against ROS-mediated cardiac injury comprises several antioxidant enzymes including SOD, catalase, and GSHPx. Among these, GSHPx is an important enzyme that performs several vital functions. It is a key antioxidant that catalyses the reduction of $\mathrm{H}_{2} \mathrm{O}_{2}$ and hydroperoxides. GSHPx not only scavenges $\mathrm{H}_{2} \mathrm{O}_{2}$, but also prevents the formation of other more toxic radicals such as $\cdot \mathrm{OH}$.
GSHPx possesses a higher affinity for $\mathrm{H}_{2} \mathrm{O}_{2}$ than catalase. Furthermore, it is present in relatively high amounts within the heart, especially in the cytosolic and mitochondrial compartments.4 ${ }^{4}$ These lines of evidence imply the primary importance of GSHPx as a defense mechanism within the heart, compared witho catalase. Moreover, GSHPx is expected to exert greater protective effects against oxidative damage than SOD because greater dismutation of $\cdot \mathrm{O}_{2}{ }^{-}$by $\mathrm{SOD}$ may result in increased $\mathrm{H}_{2} \mathrm{O}_{2}$. Therefore, compared with SOD or catalase, GSHPx is thought to be more effective in protecting cells, tissues, and organs against oxidative damage. 45

GSHPx overexpression inhibits the development of LV remodeling and failure after $\mathrm{MI}$, and so might contribute to improved survival ${ }^{46}$ These findings not only extend the previous observational study that used antioxidants, but also reveal the major role of ROS in the pathophysiology of myocardial remodeling. The effects were associated with attenuation of myocyte hypertrophy, apoptosis, and interstitial fibrosis.6 Similarly, overexpression of GSHPx attenuates myocardial remodeling and preserves diastolic function in the diabetic heart. ${ }^{77}$ Therefore, therapies designed to interfere with oxidative stress by using GSHPx could be beneficial in preventing heart failure.

\section{Peroxiredoxin-3 (Prx-3)}

We have recently demonstrated that the overexpression of a mitochondrial antioxidant, Prx-3, a member of peroxiredoxin family that can scavenge $\mathrm{H}_{2} \mathrm{O}_{2}$ in cooperation with thiol and peroxynitrite (Fig 3), protects the heart against post-MI remodeling and failure in mice. It reduces LV cavity dilatation and dysfunction, as well as myocyte hypertrophy, interstitial fibrosis, and apoptosis of the noninfarcted myocardium. These beneficial effects of Prx-3 gene overexpression are associated with attenuation of oxidative stress, mtDNA decline, and dysfunction. 48 The specific localization of Prx-3 in the mitochondria suggests that mitochondrial oxidative stress plays an important role in the development and progression of heart failure, and that the antioxidant localized specifically within the mitochondria provides a primary line of defense against this disease process.

\section{Mitochondrial Transcription Factor A (TFAM)}

TFAM is a nuclear-encoded protein that binds upstream 


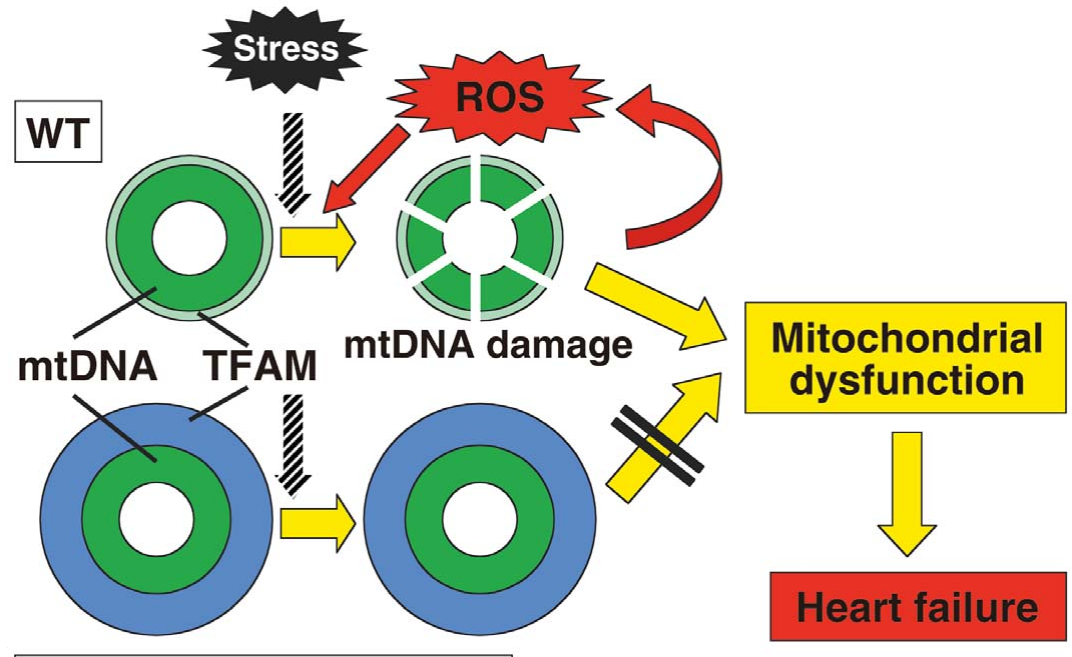

TFAM overexpression
Fig 4. Proposed mechanisms byh which mitochondrial transcription factor A (TFAM) overexpression prevents mitochondrial DNA (mtDNA) damage, oxidative stress, and myocardial remodeling and failure. In wild-type mice, TFAM directly interacts with mtDNA to form nucleoids. Stress such as ischemia causes mtDNA damage, which increases the production of reactive oxygen species and thus leads to a catastrophic cycle of mitochondrial electron transport impairment, further ROS generation, and mitochondrial dysfunction. TFAM overexpression may protect mtDNA from damage by directly binding and stabilizing mtDNA and increase the steady-state levels of mtDNA, which ameliorates mitochondrial dysfunction and thus the development and progression of heart failure. of the LSP and HSP of mtDNA and promotes transcription of mtDNA. TFAM not only regulates mtDNA transcription and replication, ${ }^{49}$ but also maintains mtDNA copy number. In fact, Tfam knockout mice, which have a 50\% reduction in their transcript and protein levels, show a $34 \%$ reduction in mtDNA copy number, $22 \%$ reduction in the mitochondrial transcript levels, and partial reduction in the cytochrome $c$ oxidase levels in the heart50 Moreover, cardiac-specific disruption in Tfam in mice results in dilated cardiomyopathy in association with a reduced amount of mtDNA and mitochondrial transcripts $5^{1}$ The transfection of antisense plasmids in culture, designed to reduce the expression of TFAM, effectively decreased the levels of mitochondrially encoded transcripts ${ }^{19}$ In contrast, forced overexpression of TFAM produced the opposite effect52 These lines of evidence obtained from knockout mice have established a critical role for TFAM in the regulation of mtDNA copy number and mitochondrial function, as well as maintenance of the physiological function of the heart in vivo. In addition, a reduction in TFAM expression has been demonstrated in several forms of cardiac failure 20,23, 3,54

By using transgenic mice that overexpress human TFAM, we examined whether TFAM could protect the heart from mtDNA deficiencies and attenuate $\mathrm{LV}$ remodeling and failure after MI55 TFAM overexpression could ameliorate the decline in mtDNA copy number and preserve it at a normal level in post-MI hearts. TFAM overexpression might increase the steady-state level of mtDNA by directly stabilizing mtDNA. Consistent with alterations in mtDNA, the decrease in oxidative capacities seen in MI was also prevented. Moreover, TFAM played an important role in myocardial protection against remodeling and failure.

Several factors may be attributed to the protective effects conferred by TFAM overexpression against myocardial remodeling and failure. First, TFAM overexpression prevented a decrease in mtDNA copy number and mitochondrial electron transport function, which may contribute to decreased myocardial oxidative stress, which in turn could contribute to the amelioration of cardiac hypertrophy, apoptosis, and interstitial fibrosis $5^{5}$ A recent study by Ekstrand et al demonstrated that the overexpression of human TFAM in the mouse increased mtDNA copy number ${ }^{56}$ These lines of evidence imply the primary importance of TFAM as a regu- latory mechanism of mtDNA copy number. TFAM has been shown to directly interact with mtDNA to form nucleoids: 57,58 Therefore, in transgenic mice increased TFAM may increase the steady-state levels of mtDNA by directly binding and stabilizing mtDNA (Fig 4). Second, TFAM overexpression may induce mitochondrial biogenesis, although this is thought to be unlikely because the number and size of the mitochondria assessed by electron microscopy were unaltered.

The results obtained from human TFAM transgenic mice differ from those from the inducible, cardiac-specific overexpression of peroxisome proliferator-activated receptor $\gamma$ coactivator $-1 \alpha$ (PGC- $1 \alpha)$ transgene in adult mice, which leads to a modest increase in mitochondrial number and the development of reversible cardiomyopathy ${ }^{5}$ PGC- $1 \alpha$ is a transcriptional coactivator and acts upstream of TFAM, and also has the capacity to increase mtDNA levels as well as mitochondrial mass in both cultured cells and transgenic mice 60,61 The reason for the discrepant results between the PGC- $1 \alpha$ and TFAM transgene overexpression studies remains unsolved, but may be related to the complex regulatory mechanisms of mitochondrial biogenesis and function by PGC- $1 \alpha$ and its downstream factors, including nuclear respiratory factors 1 and 2 and TFAM62,63

MtDNA decline and mitochondrial defects are now well recognized in a variety of diseases, such as neurodegenerative diseases, diabetes mellitus, cancer, and even aging. Therefore, with further knowledge about the mechanisms of TFAM for maintaining mtDNA copy number and mitochondrial function, it may eventually be possible to develop novel strategies for the treatment of such diseases based on manipulation of TFAM.

\section{Conclusions}

To improve the prognosis of patients with heart failure, novel therapeutic strategies based on new insights into the pathophysiology of myocardial remodeling and failure need to be developed. The approach to regulating mitochondrial oxidative stress and mtDNA damage may contribute to the establishment of effective treatment strategies for patients with heart failure. Oxidative stress is involved not only in heart failure, but also in various other diseases, including 
atherosclerosis, hypertension, and aging. Therefore, therapeutic strategies to modulate this maladaptive response should definitely become a target for future extensive investigation and could have broad application.

\section{Acknowledgments}

The work presented in this article was supported in part by grants from the Ministry of Education, Culture, Sports, Science and Technology, Japan (Nos 12670676, 14370230, 17390223, 17659223).

We thank Dr Fumiyuki Hattori for providing Prx-3 immunostaining data of cardiac myocytes.

\section{References}

1. Ho KK, Pinsky JL, Kannel WB, Levy D. The epidemiology of heart failure: The Framingham Study. J Am Coll Cardiol 1993; 22: 6A$13 \mathrm{~A}$.

2. McCord JM. Oxygen-derived free radicals in postischemic tissue injury. N Engl J Med 1985; 312: 159-163.

3. Chen H, Hu CJ, He YY, Yang DI, Xu J, Hsu CY. Reduction and restoration of mitochondrial dna content after focal cerebral ischemia/ reperfusion. Stroke 2001; 32: 2382-2387.

4. Mizuno Y, Yoshino H, Ikebe S, Hattori N, Kobayashi T, ShimodaMatsubayashi S, et al. Mitochondrial dysfunction in Parkinson's disease. Ann Neurol 1998; 44: S99-S109.

5. Trifunovic A, Wredenberg A, Falkenberg M, Spelbrink JN, Rovio AT, Bruder CE, et al. Premature ageing in mice expressing defective mitochondrial DNA polymerase. Nature 2004; 429: 417-423.

6. Belch JJ, Bridges AB, Scott N, Chopra M. Oxygen free radicals and congestive heart failure. Br Heart J 1991; 65: 245-248.

7. Hill MF, Singal PK. Antioxidant and oxidative stress changes during heart failure subsequent to myocardial infarction in rats. Am J Pathol 1996; 148: $291-300$.

8. Hill MF, Singal PK. Right and left myocardial antioxidant responses during heart failure subsequent to myocardial infarction. Circulation 1997; 96: $2414-2420$.

9. Mallat Z, Philip I, Lebret M, Chatel D, Maclouf J, Tedgui A. Elevated levels of 8-iso-prostaglandin F2alpha in pericardial fluid of patients with heart failure: A potential role for in vivo oxidant stress in ventricular dilatation and progression to heart failure. Circulation 1998; 97: 1536-1539.

10. Ide T, Tsutsui H, Kinugawa S, Suematsu N, Hayashidani S, Ichikawa $\mathrm{K}$, et al. Direct evidence for increased hydroxyl radicals originating from superoxide in the failing myocardium. Circ Res 2000; 86: $152-$ 157.

11. Tsutsui H, Ide T, Hayashidani S, Suematsu N, Utsumi H, Nakamura $\mathrm{R}$, et al. Greater susceptibility of failing cardiac myocytes to oxygen free radical-mediated injury. Cardiovasc Res 2001; 49: 103-109.

12. Ide T, Tsutsui H, Kinugawa S, Utsumi H, Kang D, Hattori N, et al. Mitochondrial electron transport complex I is a potential source of oxygen free radicals in the failing myocardium. Circ Res 1999; 85: $357-363$.

13. Sawyer DB, Colucci WS. Mitochondrial oxidative stress in heart failure: "Oxygen wastage" revisited. Circ Res 2000; 86: 119-120.

14. Munzel T, Harrison DG. Increased superoxide in heart failure: A biochemical baroreflex gone awry. Circulation 1999; 100: 216-218.

15. Bauersachs J, Bouloumie A, Fraccarollo D, Hu K, Busse R, Ertl G. Endothelial dysfunction in chronic myocardial infarction despite increased vascular endothelial nitric oxide synthase and soluble guanylate cyclase expression: Role of enhanced vascular superoxide production. Circulation 1999; 100: 292-298.

16. Attardi G, Schatz G. Biogenesis of mitochondria. Annu Rev Cell Biol 1988; 4: 289-333.

17. Shadel GS, Clayton DA. Mitochondrial DNA maintenance in vertebrates. Annu Rev Biochem 1997; 66: 409-435.

18. Clayton DA. Replication and transcription of vertebrate mitochondrial DNA. Annu Rev Cell Biol 1991; 7: 453-478.

19. Kajander OA, Karhunen PJ, Jacobs HT. The relationship between somatic mtDNA rearrangements, human heart disease and aging. Hum Mol Genet 2002; 11: 317-324.

20. Lebrecht D, Setzer B, Ketelsen UP, Haberstroh J, Walker UA. Timedependent and tissue-specific accumulation of mtDNA and respiratory chain defects in chronic doxorubicin cardiomyopathy. Circulation 2003; 108: 2423-2429.

21. Naya FJ, Black BL, Wu H, Bassel-Duby R, Richardson JA, Hill JA, et al. Mitochondrial deficiency and cardiac sudden death in mice lacking the MEF2A transcription factor. Nat Med 2002; 8: 1303-1309.

22. Wallace DC. Mitochondrial diseases in man and mouse. Science
1999; 283: $1482-1488$.

23. Ide T, Tsutsui H, Hayashidani S, Kang D, Suematsu N, Nakamura K, et al. Mitochondrial DNA damage and dysfunction associated with oxidative stress in failing hearts after myocardial infarction. Circ Res 2001; 88: 529-535.

24. Giulivi C, Boveris A, Cadenas E. Hydroxyl radical generation during mitochondrial electron transfer and the formation of 8-hydroxydesoxyguanosine in mitochondrial DNA. Arch Biochem Biophys 1995; 316: $909-916$.

25. Williams RS. Mitochondrial gene expression in mammalian striated muscle: Evidence that variation in gene dosage is the major regulatory event. J Biol Chem 1986; 261: 12390-12394.

26. Ballinger SW, Patterson C, Yan CN, Doan R, Burow DL, Young CG, et al. Hydrogen peroxide- and peroxynitrite-induced mitochondrial DNA damage and dysfunction in vascular endothelial and smooth muscle cells. Circ Res 2000; 86: 960-966.

27. Williams RS. Canaries in the coal mine: Mitochondrial DNA and vascular injury from reactive oxygen species. Circ Res 2000; 86: $915-$ 916.

28. Anan R, Nakagawa M, Miyata M, Higuchi I, Nakao S, Suehara M, et al. Cardiac involvement in mitochondrial diseases: A study on 17 patients with documented mitochondrial DNA defects. Circulation 1995; 91: 955-961.

29. Corral-Debrinski M, Shoffner JM, Lott MT, Wallace DC. Association of mitochondrial DNA damage with aging and coronary atherosclerotic heart disease. Mutat Res 1992; 275: 169-180.

30. Arbustini E, Diegoli M, Fasani R, Grasso M, Morbini P, Banchieri N, et al. Mitochondrial DNA mutations and mitochondrial abnormalities in dilated cardiomyopathy. Am J Pathol 1998; 153: 1501-1510.

31. Clayton DA, Williams RS, Liang I. Meeting highlights. Circulation 1995; 92: 2022-2023.

32. Spinale FG, Coker ML, Thomas CV, Walker JD, Mukherjee R, Hebbar L. Time-dependent changes in matrix metalloproteinase activity and expression during the progression of congestive heart failure: Relation to ventricular and myocyte function. Circ Res 1998; 82: $482-495$.

33. Siwik DA, Tzortzis JD, Pimental DR, Chang DL, Pagano PJ, Singh $\mathrm{K}$, et al. Inhibition of copper-zinc superoxide dismutase induces cell growth, hypertrophic phenotype, and apoptosis in neonatal rat cardiac myocytes in vitro. Circ Res 1999; 85: 147-153.

34. Creemers EE, Cleutjens JP, Smits JF, Daemen MJ. Matrix metalloproteinase inhibition after myocardial infarction: A new approach to prevent heart failure? Circ Res 2001; 89: 201-210.

35. Rohde LE, Ducharme A, Arroyo LH, Aikawa M, Sukhova GH, Lopez-Anaya A, et al. Matrix metalloproteinase inhibition attenuates early left ventricular enlargement after experimental myocardial infarction in mice. Circulation 1999; 99: 3063-3070.

36. Hayashidani S, Tsutsui H, Ikeuchi M, Shiomi T, Matsusaka H, Kubota T, et al. Targeted deletion of MMP-2 attenuates early LV rupture and late remodeling after experimental myocardial infarction. Am J Physiol Heart Circ Physiol 2003; 285: H1229-H1235.

37. Rajagopalan S, Meng XP, Ramasamy S, Harrison DG, Galis ZS. Reactive oxygen species produced by macrophage-derived foam cells regulate the activity of vascular matrix metalloproteinases in vitro: Implications for atherosclerotic plaque stability. J Clin Invest 1996; 98: $2572-2579$.

38. Kinugawa S, Tsutsui H, Hayashidani S, Ide T, Suematsu N, Satoh S, et al. Treatment with dimethylthiourea prevents left ventricular remodeling and failure after experimental myocardial infarction in mice: Role of oxidative stress. Circ Res 2000; 87: 392-398.

39. Sullivan MJ, Green HJ, Cobb FR. Skeletal muscle biochemistry and histology in ambulatory patients with long-term heart failure. Circulation 1990; 81: 518-527.

40. Wilson JR. Exercise intolerance in heart failure: Importance of skeletal muscle. Circulation 1995; 91: 559-561.

41. Nishiyama Y, Ikeda H, Haramaki N, Yoshida N, Imaizumi T. Oxidative stress is related to exercise intolerance in patients with heart failure. Am Heart J 1998; 135: 115-120.

42. Tsutsui H, Ide T, Hayashidani S, Suematsu N, Shiomi T, Wen J, et al. Enhanced generation of reactive oxygen species in the limb skeletal muscles from a murine infarct model of heart failure. Circulation 2001; 104: 134-136.

43. Kinugawa S, Wang Z, Kaminski PM, Wolin MS, Edwards JG, Kaley $\mathrm{G}$, et al. Limited exercise capacity in heterozygous manganese superoxide dismutase gene-knockout mice: Roles of superoxide anion and nitric oxide. Circulation 2005; 111: 1480-1486.

44. Le CT, Hollaar L, van der Valk EJ, van der Laarse A. Buthionine sulfoximine reduces the protective capacity of myocytes to withstand peroxide-derived free radical attack. J Mol Cell Cardiol 1993; 25: $519-528$. 
45. Toussaint O, Houbion A, Remacle J. Relationship between the critical level of oxidative stresses and the glutathione peroxidase activity. Toxicology 1993; 81: 89-101.

46. Shiomi T, Tsutsui H, Matsusaka H, Murakami K, Hayashidani S, Ikeuchi M, et al. Overexpression of glutathione peroxidase prevents left ventricular remodeling and failure after myocardial infarction in mice. Circulation 2004; 109: 544-549.

47. Matsushima S, Kinugawa S, Ide T, Matsusaka H, Inoue N, Ohta Y, et al. Overexpression of glutathione peroxidase attenuates myocardial remodeling and preserves diastolic function in diabetic heart. Am J Physiol Heart Circ Physiol 2006; 291: H2237-H2245.

48. Matsushima S, Ide T, Yamato M, Matsusaka H, Hattori F, Ikeuchi M, et al. Overexpression of mitochondrial peroxiredoxin-3 prevents left ventricular remodeling and failure after myocardial infarction in mice. Circulation 2006; 113: 1779-1786.

49. Scarpulla RC. Nuclear activators and coactivators in mammalian mitochondrial biogenesis. Biochim Biophys Acta 2002; 1576: 1-14.

50. Larsson NG, Wang J, Wilhelmsson H, Oldfors A, Rustin P, Lewandoski M, et al. Mitochondrial transcription factor A is necessary for mtDNA maintenance and embryogenesis in mice. Nat Genet 1998; 18: $231-236$.

51. Wang J, Wilhelmsson H, Graff C, Li H, Oldfors A, Rustin P, et al. Dilated cardiomyopathy and atrioventricular conduction blocks induced by heart-specific inactivation of mitochondrial DNA gene expression. Nat Genet 1999; 21: 133-137.

52. Montoya J, Perez-Martos A, Garstka HL, Wiesner RJ. Regulation of mitochondrial transcription by mitochondrial transcription factor A. Mol Cell Biochem 1997; 174: 227-230.

53. Garnier A, Fortin D, Delomenie C, Momken I, Veksler V, VenturaClapier R. Depressed mitochondrial transcription factors and oxidative capacity in rat failing cardiac and skeletal muscles. $J$ Physiol 2003; 551: $491-501$.

54. Kanazawa A, Nishio Y, Kashiwagi A, Inagaki H, Kikkawa R, Horiike $\mathrm{K}$. Reduced activity of mtTFA decreases the transcription in mito- chondria isolated from diabetic rat heart. Am J Physiol Endocrinol Metab 2002; 282: E778-E785.

55. Ikeuchi M, Matsusaka H, Kang D, Matsushima S, Ide T, Kubota T, et al. Overexpression of mitochondrial transcription factor a ameliorates mitochondrial deficiencies and cardiac failure after myocardial infarction. Circulation 2005; 112: 683-690.

56. Ekstrand MI, Falkenberg M, Rantanen A, Park CB, Gaspari M, Hultenby $\mathrm{K}$, et al. Mitochondrial transcription factor A regulates mtDNA copy number in mammals. Hum Mol Genet 2004; 13: $935-$ 944.

57. Alam TI, Kanki T, Muta T, Ukaji K, Abe Y, Nakayama H, et al. Human mitochondrial DNA is packaged with TFAM. Nucleic Acids Res 2003; 31: 1640-1645.

58. Takamatsu C, Umeda S, Ohsato T, Ohno T, Abe Y, Fukuoh A, et al. Regulation of mitochondrial D-loops by transcription factor $\mathrm{A}$ and single-stranded DNA-binding protein. EMBO Rep 2002; 3: 451 456.

59. Russell LK, Mansfield CM, Lehman JJ, Kovacs A, Courtois M, Saffitz JE, et al. Cardiac-specific induction of the transcriptional coactivator peroxisome proliferator-activated receptor gamma coactivator-1alpha promotes mitochondrial biogenesis and reversible cardiomyopathy in a developmental stage-dependent manner. Circ Res 2004; 94: 525-533

60. Lin J, Wu H, Tarr PT, Zhang CY, Wu Z, Boss O, et al. Transcriptional co-activator PGC-1 alpha drives the formation of slow-twitch muscle fibres. Nature 2002; 418: 797-801.

61. Wu Z, Puigserver P, Andersson U, Zhang C, Adelmant G, Mootha V, et al. Mechanisms controlling mitochondrial biogenesis and respiration through the thermogenic coactivator PGC-1. Cell 1999; 98: $115-124$.

62. Huss JM, Kelly DP. Nuclear receptor signaling and cardiac energetics. Circ Res 2004; 95: 568-578.

63. Ventura-Clapier R, Garnier A, Veksler V. Energy metabolism in heart failure. J Physiol 2004; 555: 1-13. 